\title{
Wave propagation in a solar quiet region and the influence of the magnetic canopy
}

\author{
I. Kontogiannis, G. Tsiropoula, and K. Tziotziou
}

\author{
Institute for Astronomy, Astrophysics, Space Applications and Remote Sensing, National Observatory of Athens, \\ 15236 Penteli, Greece \\ e-mail: [jkonto;georgia;kostas]@noa.gr
}

Received 24 July 2015 / Accepted 20 November 2015

\begin{abstract}
Aims. We seek indications or evidence of transmission/conversion of magnetoacoustic waves at the magnetic canopy, as a result of its impact on the properties of the wave field of the photosphere and chromosphere.

Methods. We use cross-wavelet analysis to measure phase differences between intensity and Doppler signal oscillations in the $\mathrm{H} \alpha$, $\mathrm{Ca}$ II H, and $G$-band. We use the height of the magnetic canopy to create appropriate masks to separate internetwork (IN) and magnetic canopy regions. We study wave propagation and differences between these two regions.

Results. The magnetic canopy affects wave propagation by lowering the phase differences of progressive waves and allowing the propagation of waves with frequencies lower than the acoustic cut-off. We also find indications in the Doppler signals of H $\alpha$ of a response to the acoustic waves at the IN, observed in the CaII H line. This response is affected by the presence of the magnetic canopy.

Conclusions. Phase difference analysis indicates the existence of a complicated wave field in the quiet Sun, which is composed of a mixture of progressive and standing waves. There are clear imprints of mode conversion and transmission due to the interaction between the $p$-modes and small-scale magnetic fields of the network and internetwork.
\end{abstract}

Key words. Sun: chromosphere - Sun: photosphere - Sun: oscillations - Sun: magnetic fields

\section{Introduction}

Wave propagation has been a long standing field of research in solar physics, spanning several decades of literature. The discovery of the "5-min oscillations" by Leighton et al. (1962) and the observation and study of the $p$-mode spectrum using " $\mathrm{k}-\omega$ " diagrams by Deubner (1975) and Deubner et al. (1979) showed that there is a pool of acoustic oscillations, with periods of a few minutes that permeate the solar interior and atmosphere. These acoustic oscillations are capable of transporting and depositing energy and may also give valuable information on local physical conditions. Several aspects concerning wave propagation are established through a series of papers (e.g. Mein \& Mein 1976; Lites \& Chipman 1979; Lites et al. 1982; Kneer \& von Uexküll 1985; Fleck 1989; Deubner \& Fleck 1989, 1990; Deubner et al. 1990; Lites et al. 1993; Krijger et al. 2001). One of the tools used for diagnosing the details of wave propagation is phase difference analysis, which facilitates the comparison of velocity and intensity signals that form at the same or different atmospheric layers. In the traditional view of wave propagation in the solar atmosphere, waves with frequencies lower than $5.2 \mathrm{mHz}$ cannot propagate from the photosphere to the chromosphere. This theoretical property has been verified by the small phase differences measured between oscillations at two heights. Currently, owing to high-resolution observations, this description is being reassessed to include wave propagation with respect to the local magnetic field topology, since the latter is playing a crucial role in wave processes at the solar atmosphere.

In contrast to its definition, quiet Sun continuously and ubiquitously exhibits a large range of dynamic phenomena associated with small scale, albeit intense, magnetic field concentrations.
Pushed by convective and near surface motions, magnetic fields accumulate at the boundaries of supergranules and form the magnetic network, visible as a bright web-like pattern (in filtergrams of strong chromospheric lines such as $\mathrm{Ca}$ II H) surrounding larger areas of very low magnetic flux called internetwork (IN). These bright points betray, in fact, the presence of magnetic flux tubes, which expand with height, as the ambient atmospheric pressure drops, and result in highly inclined magnetic fields that dominate the dynamics of the overlying chromosphere. Observationally, in the $\mathrm{H} \alpha$ line $(6563 \AA)$ this magnetic configuration manifests itself through the ubiquitous presence of elongated absorption features, called mottles (Beckers 1968; Tsiropoula et al. 2012), which stem from the network and partially cover the IN. Mottles form the so-called magnetic canopy (Gabriel 1976; Kontogiannis et al. 2010b), which roughly separates the stratified, gas-pressure dominated environment of the photosphere and lower chromosphere and the plasma of the upper atmosphere, which is highly structured by the magnetic field. The plasma- $\beta$, denoting the ratio of the gas pressure to the magnetic pressure, is used to parameterize the plasma dynamics, while the height at which $\beta=1$ defines the height of the magnetic canopy.

The interaction of the acoustic oscillations with the magnetic field of the network and the magnetic canopy has been illustrated in a series of studies. In general, the magnetic field may allow or inhibit the propagation of waves of certain frequencies. The presence of both intense and inclined magnetic field may lower the acoustic cut-off frequency, allowing the propagation of waves with frequencies lower than $5.2 \mathrm{mHz}$ (Michalitsanos 1973; Bel \& Leroy 1977; Suematsu 1990); this may explain 
the detection of $5 \mathrm{~min}$ oscillations in chromospheric mottles, which is a common finding (Tziotziou et al. 2004; Tsiropoula et al. 2009). The chromospheric magnetic field is also responsible for the so-called "power halos" and "magnetic shadows", areas of increased and suppressed power, respectively, found over and around the network (Krijger et al. 2001; Judge et al. 2001; McIntosh \& Judge 2001; McIntosh et al. 2003; Vecchio et al. 2007; Reardon et al. 2009; Kontogiannis et al. 2010a). This was also clearly demonstrated in Kontogiannis et al. (2010b, 2014) who concluded that waves reflect and refract on the magnetic canopy, increasing the power below and decreasing it above.

Details of the mechanism that produces wave reflection and refraction on the magnetic canopy have been highlighted in several theoretical works and simulations with increasing level of sophistication (Rosenthal et al. 2002; Bogdan et al. 2003; Carlsson 2006; Khomenko \& Collados 2006, 2009; Khomenko et al. 2008; Nutto et al. 2012). Following these studies, it is clear that the critical parameter for the interaction between the acoustic waves and the magnetic canopy is the attack angle, defined by the inclination of the magnetic field vector and the direction of the wave-vector. Upon hitting the magnetic canopy, the acoustic waves transfer part of their energy to a slow magnetoacoustic wave (a process termed transmission) and part to a fast magnetoacoustic wave (a process termed conversion). The amount of energy that goes to each of these modes has been defined in the model of Schunker \& Cally (2006) and Cally (2007) and depends on the attack angle, the frequency of the wave, and the thickness of the magnetic canopy. Application on solar observations explains very well the distribution of acoustic power around sunspots (Stangalini et al. 2011) and the network (Kontogiannis et al. 2014).

As a consequence of this kind of interaction, waves in the chromosphere are strongly guided by the magnetic field. Slow waves travel along the magnetic field lines, while fast waves propagate perpendicularly and eventually reflect, propagating downwards and forming standing waves. This sequence of events probably results to a complicated wave field, since standing and propagating waves (both vertically and obliquely) are superposed to a different extent, depending on the height and position in the solar atmosphere. In this paper we measure the phase differences of oscillations using time series of filtergrams that represent different atmospheric heights and dynamic regimes. Our aim is to examine whether the differences between the IN and the magnetic canopy, if any, are consistent with the scheme of transmission and conversion of magnetoacoustic waves on the magnetic canopy.

\section{Observations}

Our data were obtained on October 15, 2007, as part of an observational campaign, which included several ground-based and space-born instruments. We used data from the Dutch Open Telescope (DOT; Rutten et al. 2004a) and the Spectropolarimeter of the Solar Optical Telescope (SOT/SP; Tsuneta et al. 2008) on board Hinode (Kosugi et al. 2007).

The DOT observed a $84^{\prime \prime} \times 87^{\prime \prime}$ area located at the centre of the solar disk between 08:32-09:53 UT and provided a time series of speckle reconstructed images in five positions along the $\mathrm{H} \alpha$ line profile (line centre, $\pm 0.35 \AA, \pm 0.70 \AA$ ), as well as $\mathrm{Ca}$ II H and $G$-band filtergrams. The cadence of the time series is $30 \mathrm{~s}$ and the spatial resolution is $0.109 \mathrm{arcsec} / \mathrm{pixel}$. The spectral coverage of the $\mathrm{H} \alpha$ profile is implemented throughout the $30 \mathrm{~s}$ run and, therefore, images at different wavelengths of the profile have been acquired with a small time difference. To compensate for this effect, we spline-interpolated all filtergrams to a common time axis and in the following we consider that they were all acquired simultaneously.

The intensities at $\pm 0.35 \AA$ and $\pm 0.70 \AA$ from the $\mathrm{H} \alpha$ line centre, are used to calculate the corresponding Doppler signals (DS), through the formula

$\mathrm{DS}=\frac{I(+\Delta \lambda)-I(-\Delta \lambda)}{I(+\Delta \lambda)+I(-\Delta \lambda)}$.

The DS gives a parametric description of the velocity with upwards motion denoted by positive values and vice versa. Also, the averaged wing intensity may be calculated by averaging the intensity at opposite wings, i.e.

$I_{\mathrm{avg}}(\Delta \lambda)=\frac{I(+\Delta \lambda)+I(-\Delta \lambda)}{2}$.

This gives a more reliable intensity measure at the corresponding wing position, compensated for the effect of Doppler shift.

The SOT/SP performed a raster scan of the same region in the Fe I 6301.5 and $6302.5 \AA$ lines (in normal mode), with a spatial resolution of $0.32 \mathrm{arcsec} /$ pixel between 09:05-09:15 UT. The corresponding vector magnetogram was produced by the Community Spectropolarimetric Analysis Center of the High Altitude Observatory (HAO/CSAC), through inversion of the Stokes spectra via the MERLIN code. Further details on the observations, the speckle reconstruction procedure of the $\mathrm{H} \alpha$ observations, data reduction steps, and the inversion of the Stokes spectra can be found in Rutten et al. (2004a) and Kontogiannis et al. (2010b).

Standard data reduction procedures were used, with a considerable effort devoted to image co-alignment, through crosscorrelation, between average images at different filters (Fig. 1). In the common field of view (FOV) of $36^{\prime \prime} \times 44^{\prime \prime}$ we studied, a well-defined rosette of mottles is found around a network boundary cluster of positive polarity magnetic fields (Fig. 1). Since we are planning to study wave propagation inside certain areas of the FOV, a few limitations arise: the examined time series must have no gaps in image acquisition and must be co-temporal with high-resolution magnetograms. The part of the time series that satisfies both conditions is between 09:00-09:30 UT.

\section{Analysis}

\subsection{Determination of the height of the magnetic canopy}

The SOT/SP raster was used as the lower photospheric boundary condition to calculate the chromospheric magnetic field through a current-free (potential field) extrapolation (Schmidt 1964) up to $2500 \mathrm{~km}$. The potential extrapolation gives the vector magnetic field at the chromosphere, which corresponds to the minimum current-free energy state (for details see Kontogiannis et al. $2010 \mathrm{~b}, 2011)$. Then the plasma- $\beta$ parameter $\left(\beta=P_{\mathrm{gas}} / P_{\mathrm{mag}}\right)$ is calculated, where $P_{\text {gas }}$, the gas pressure, is taken from model C of Vernazza et al. (1981) and $P_{\text {mag }}$ is the magnetic pressure $\left(B^{2} / 2 \mu_{0}\right)$. The height of the magnetic canopy was then determined, as the height where $\beta$ is of order unity (see Kontogiannis et al. 2010b).

To study the effect of the magnetic canopy on wave propagation, the FOV was divided in two parts, using the height of the magnetic canopy as a criterion. We chose this classification based on the comparison between the height of the magnetic canopy and the power maps of 3 and 5 min acoustic oscillations from Kontogiannis et al. (2010b). We consider as 


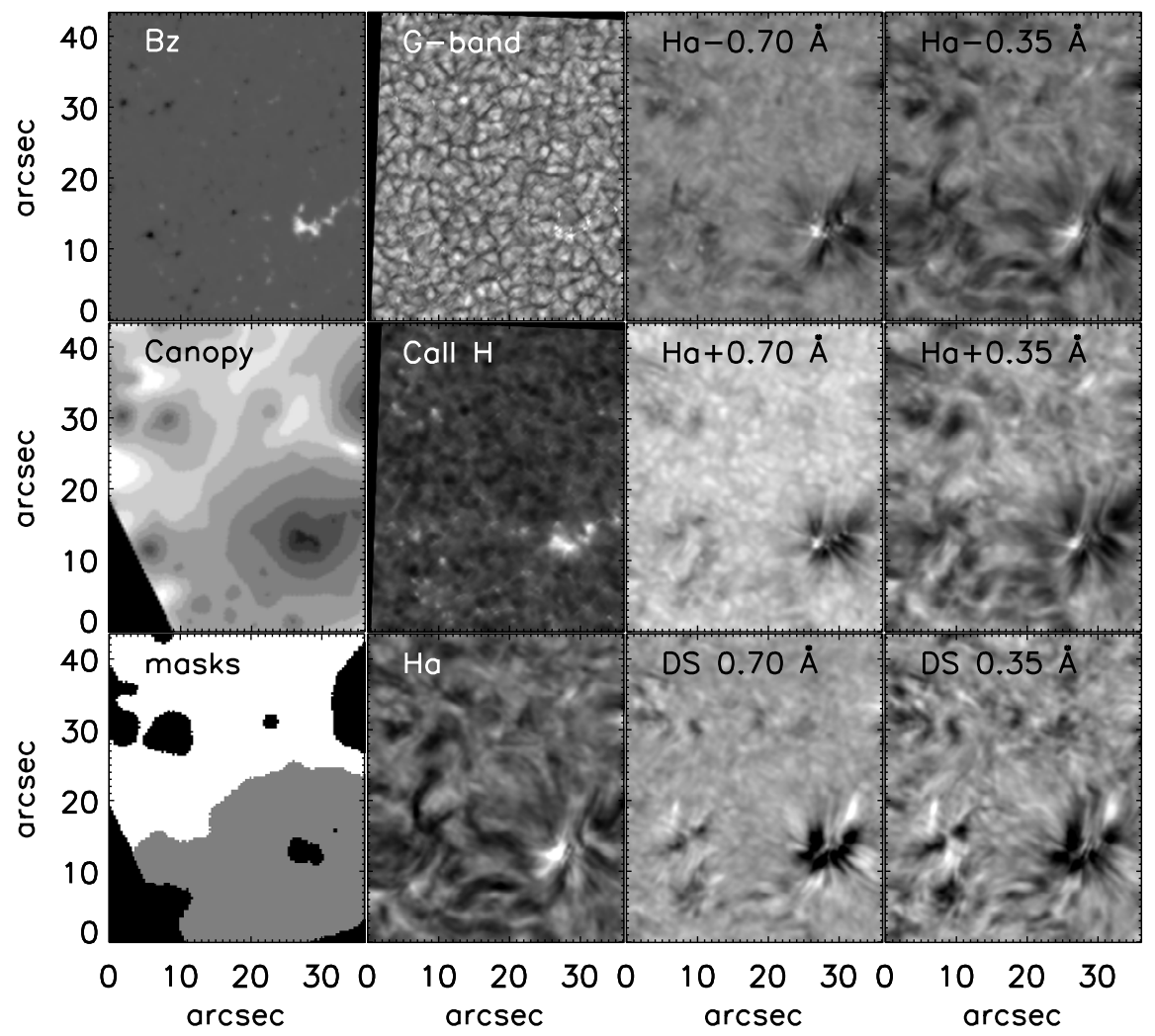

Fig. 1. Summary of the observations of the quiet Sun area under study. In the left panel, top row, white denotes positive polarity. In the left panel, middle row, lighter shading denotes higher canopy while canopy heights start at $250 \mathrm{~km}$ and increase in $235 \mathrm{~km}$ steps. In the left panel, bottom row, white denotes the mask of the IN area, grey the mask of the canopy area while the black regions have not been taken under consideration. Images and DS maps are temporal averages. At the DS maps of the bottom row, white denotes upwards and black denotes downwards motion.

"IN" the part of the FOV where the magnetic canopy is higher than $1600 \mathrm{~km}$ and as "canopy", the area around the well-formed rosette, where the height of the magnetic canopy is between $720 \mathrm{~km}$ and $1600 \mathrm{~km}$. We have excluded from our analysis the network boundaries, where the magnetic canopy forms lower than $720 \mathrm{~km}$ as well as some low-lying canopies found at the IN (Fig. 1).

\subsection{Phase difference analysis}

We examine wave propagation at the IN and the magnetic canopy through a cross-wavelet transform (Torrence \& Compo 1998) between intensities, DS, or intensity-DS pairs on every pixel of the FOV. Although phase differences may be obtained with a fast Fourier transform (widely utilized in past studies), wavelet analysis was chosen as a more suitable method because of the intermittency exhibited by the solar oscillations. We used the Morlet wavelet function, which is a sinusoid modulated by a Gaussian and is appropriate and commonly used for harmonictype oscillations.

While a large part of the literature has been based on the " $\mathrm{k}-\omega$ " diagrams, including studies on the global nature of solar oscillations persisting up to chromospheric heights (Kneer \& von Uexküll 1985; Deubner et al. 1996), for reasons of consistency with our previous works and to facilitate comparison between results, our analysis is restricted only to frequencies. This approach has been widely used in nominal works referring to network-IN oscillations (e.g. Mein \& Mein 1976; Lites \& Chipman 1979; Lites et al. 1982; Deubner et al. 1990; Lites et al. 1993). All of these studies examined the vertical propagation of acoustic waves. This, of course, does not mean that acoustic waves propagate only vertically. In the last section, we will discuss the limitations posed by this assumption and the kind of analysis and data sets needed to overcome these limitations. Thus, in the present analysis we focus on the interaction between the vertically propagating acoustic waves with the inclined magnetic field lines that form the magnetic canopy in an attempt to provide further supporting evidence to our findings regarding the role of the magnetic canopy in the transmission/conversion of magnetoacoustic waves at the network/IN areas (Kontogiannis et al. 2014).

A cross-wavelet analysis of two signals gives the crosspower, coherence, and phase difference as functions of time and period (frequency). Coherence is a measure of the cross-correlation between the two time series and assumes values from zero (where no correlation exists between the two time series) to unity. Random noise, however, produces a non-zero coherence and therefore, a coherence threshold must be determined, above which the phase difference is considered reliable. This task is not trivial in the case of the wavelet transform. In order to remedy this, we adopt the "floor exceedance coherency approach" described in Bloomfield et al. (2004). The resulting coherence threshold for all time series is frequency dependent and, in most cases, it is around 0.6.

We discarded all phase differences that correspond to lower coherence values. For the remaining phase differences, we follow the methodology of Lites \& Chipman (1979) by constructing halftone images, where the crosspower at every pixel of the area is summed over $3^{\circ}$ wide bins of phase difference for each frequency element (calculated for the periods of the wavelet transform). To improve the readability of these images, 
the crosspower distribution for each frequency is normalized to unity.

We should stress that through this methodology the phase differences themselves are not averaged inside the corresponding areas. Instead, the resulting 2D graphs represent the distribution of the crosspower measured in each pixel of the canopy/IN areas, frequency, and phase difference bins, and these 2D graphs serve two purposes. First, they exhibit the trend of phase difference as a function of frequency. To further enhance this feature, we also calculated the position of the maximum crosspower for each frequency and overlaid its position on each distribution. Second, the width of the distribution gives a measure of the scatter of crosspower (Lites et al. 1993). To provide a measure of this scatter, we overlay the $50 \%$ contour of the halftone images as an estimate for the phase difference distribution width (representing the FWHM of the crosspower distribution at each frequency). The difference in our approach, compared to previous studies, is that we utilize all available crosspower inside the canopy/IN areas to construct the halftone images, provided that it corresponds to coherence higher than the previously derived threshold.

For vertical wave propagation between two atmospheric levels and for frequencies higher than the acoustic cut-off, the dependence of phase difference on frequency is given by:

$\Delta \phi=2 \pi \frac{\mathrm{d} h}{v_{\mathrm{ph}}} f$

where $f$ is the frequency, $v_{\text {ph }}$ the phase speed, and $\mathrm{d} h$ the height separation between the height of formation (HOF) of the considered intensity/velocity pair. Therefore, when measuring phase differences between two fixed heights, a monotonic increase of phase differences is an indication of upwards vertical propagation. For a given height separation, the steeper the gradient, the lower the phase speed, and one may use the gradient to determine the average phase speed. On the other hand, small and constant phase differences are an indication of standing or evanescent waves. The interpretation of phase differences between intensity and velocity signals within the same spectral line is more complicated because of the necessary simplifications adopted concerning the spectral line formation details and the dependence of brightness on the thermodynamic parameters of the emitting/absorbing gas (i.e. temperature,density). Several theoretical studies have addressed this issue in the past and we attempt to utilize their results.

\section{Results}

\subsection{Phase differences between Ca II H and G-band}

First, we examine wave propagation between the photosphere in the $G$-band and the layers sampled by the $\mathrm{Ca}$ II $\mathrm{H}$ bandpass. This also gives us the opportunity to test our approach since this pair has been studied before (see e.g. Rutten et al. 2004b; Lawrence \& Cadavid 2012). The wide bandpass of the Ca II H filter contains contribution from the core of the line, which is of chromospheric origin. This contribution, however, is overshadowed by the photospheric part of the line (Reardon et al. 2009) and, therefore, the bandpass mostly shows the reversed granulation and the network boundary. The DOT Ca II H intensity is estimated by Rutten et al. (2004b) to reflect heights around $250 \mathrm{~km}$. On the other hand, the $G$-band is formed up to a few tens of km above $\tau_{5000}=1$, so it is reasonable to assume a height separation between the two layers of around $200 \mathrm{~km}$.

Figure 2 shows the phase differences between the two bandpasses at the IN and the canopy. In line with previous studies, both at the IN and the canopy regions, the negative phase differences at the low-frequency range (below $3 \mathrm{mHz}$ ) are attributed to gravity waves (Mihalas \& Toomre 1981) and the intensity anti-correlation between $\mathrm{Ca}$ II $\mathrm{H}$ and $G$-band due to the reversed granulation.

At the IN (Fig. 2, left panel), phase differences between $3 \mathrm{mHz}$ and $5 \mathrm{mHz}$ are constant and around $0^{\circ}$, indicating the presence of evanescent waves. Then, they start to increase at $\sim 4.5 \mathrm{mHz}$, while the width of the distribution shows significant cross-power corresponding to positive phase difference at frequencies as low as $3 \mathrm{mHz}$. The inference of a cut-off frequency lower than $5.2 \mathrm{mHz}$ at the IN is an interesting finding, suggesting the presence of small-scale, unresolved inclined magnetic fields. This is in agreement with the latest observations that reveal magnetic fields at the IN, stronger than previously thought (Lites et al. 2008). Indeed, an inspection of the original SOT/SP raster magnetogram shows a multitude of small-scale LOS magnetic elements at the IN with measured strengths around $100 \mathrm{G}$. The presence of strong magnetic fields in very small scales cannot be ruled out because these fields would be smeared by the resolution element, since magnetic flux, instead of the field strength, is measured. However, even in the absence of strong magnetic fields, a lower cut-off value is possible as the result of radiative damping in an isothermal atmosphere (see e.g. Worrall 2002). Above $5 \mathrm{mHz}$, phase differences increase monotonically with frequency. A linear fit on the maxima of the distribution leads to an estimation of the phase speed at $6.6 \pm 0.2 \mathrm{~km} \mathrm{~s}^{-1}$, which is compatible with the sound speed at the photosphere.

At the magnetic canopy (Fig. 2, right panel), phase differences start increasing below $4 \mathrm{mHz}$. This is an indication of the lowering of the acoustic cut-off frequency in the vicinity of the network boundaries (Michalitsanos 1973). Phase differences increase with frequency, above $4 \mathrm{mHz}$, as expected for acoustic wave propagation. The gradient of the distribution is lower than that at the IN and leads to a higher phase speed $\left(10.3 \pm 0.7 \mathrm{~km} \mathrm{~s}^{-1}\right)$. A higher phase speed in the vicinity of the magnetic canopy is explained on the grounds of mode conversion according to which part of the energy carried by the acoustic waves is transferred to the fast magneto-acoustic mode. The fast speed increases near the network boundary, as the strength of the magnetic field increases there as well.

Lower phase differences near the network boundary may also be produced by the decrease of the height separation between the two layers as a result of the presence of the diverging magnetic field. This scenario was invoked by McIntosh \& Smillie (2004) to explain the dependence of wave packet characteristics on the magnetic field strength. Nutto et al. (2012), using realistic MHD simulations of the quiet Sun, conclude that indeed it is the combination of this effect with the increasing phase speed of the fast mode due to the presence of the magnetic canopy, which produces shortened travel times, i.e. lower phase differences. The suppression of acoustic power around magnetic concentrations in Ca II H observations (Lawrence \& Cadavid 2010) shows that the magnetic canopy affects the oscillatory field in this bandpass and, therefore, the latter mechanism may also be in effect as well.

The effect of the magnetic canopy in our data is not so pronounced as in the case of Lawrence \& Cadavid (2012). The reason lies in our mask selection and the fact that in our FOV, there is a highly localized network boundary instead of an extended distribution of bright points. Lawrence \& Cadavid examine phase differences at the part of their FOV where the canopy is located below $400 \mathrm{~km}$, whereas we examine the areas where the canopy is located below $1600 \mathrm{~km}$. Given the fact that the 

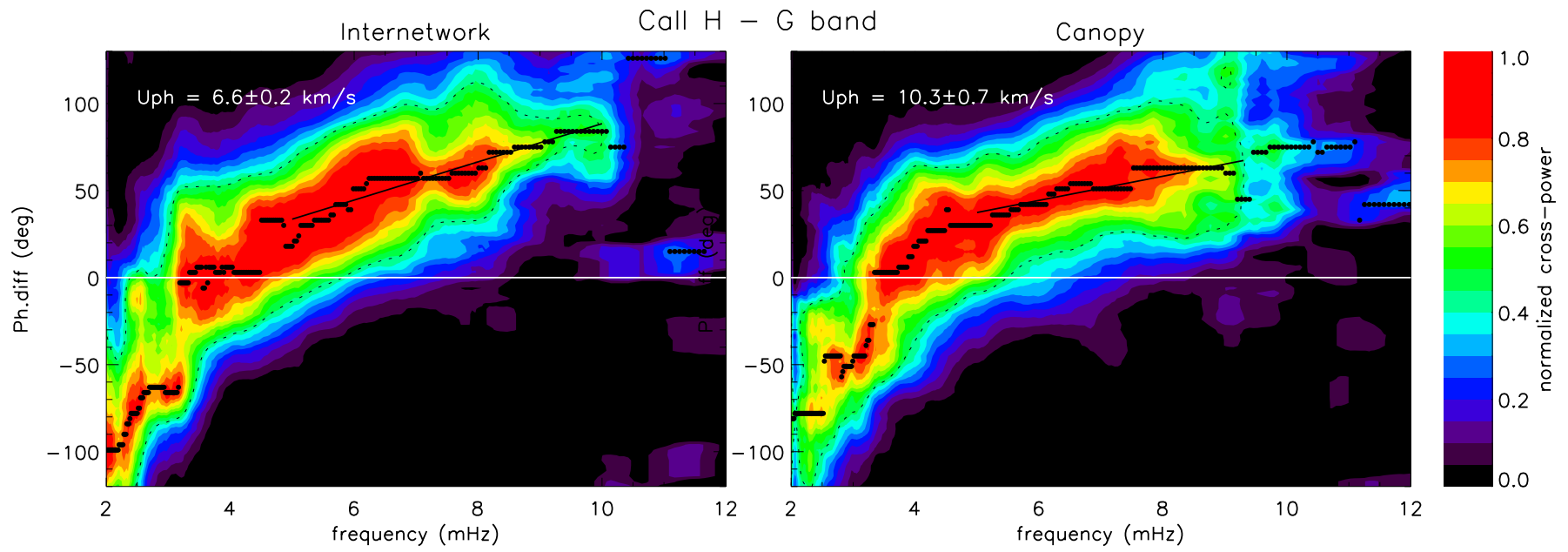

Fig. 2. Phase differences between Ca II H-G-band at the IN (left) and the magnetic canopy (right). Filled contours represent the crosspower distribution, black points denote the positions of maximum crosspower, normalized to unity for each frequency element, and the dashed contour indicates the 50\% level from maximum crosspower (as an indicator of FWHM). Crosspower below 10\% has been disregarded. Also, overplotted are regression lines calculated above $5.2 \mathrm{mHz}$ up to the part of the distribution enclosed by the 0.5 contour. From their gradient, the corresponding phase velocities are calculated, assuming that the height separation between Ca II H and $G$-band is $200 \mathrm{~km}$ (see text).
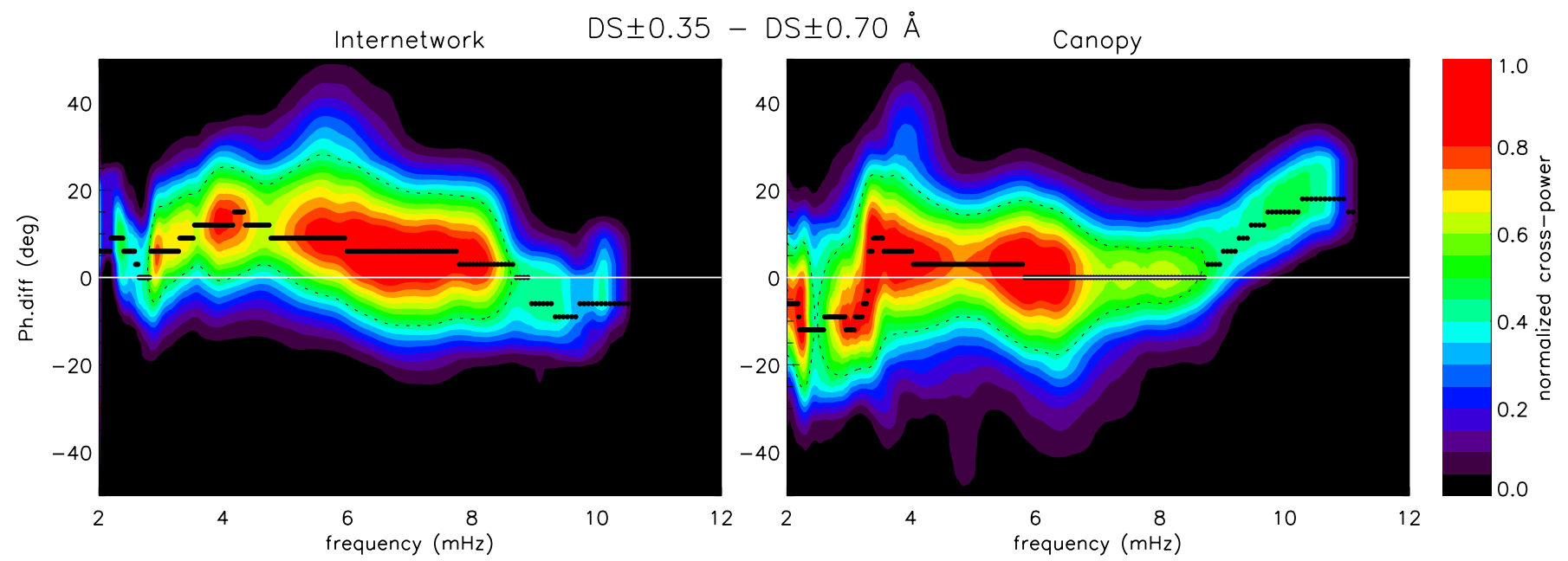

Fig. 3. Same as Fig. 2, for the pair $\mathrm{H} \alpha \mathrm{DS}$ at $\pm 0.35 \AA$ and $\pm 0.70 \AA$.

$\mathrm{Ca}$ II $\mathrm{H}$ line forms below the temperature minimum, within our canopy region there is increased contribution from the undisturbed IN. In fact, examining the phase differences at different canopy heights, the outcome is a distribution similar to those in Fig. 2, but with decreasing inclination and, therefore, our findings are in line with previous results.

\subsection{Phase differences between $\mathrm{H} \alpha$ Doppler signals}

According to Leenaarts et al. (2006), the $\mathrm{H} \alpha$ wing at $\pm 0.70 \AA$ is of photospheric origin $(200-600 \mathrm{~km})$, while the $\pm 0.35 \AA$ is mostly chromospheric $(800-1600 \mathrm{~km})$, and also contains a significant photospheric contribution. This makes the $\mathrm{H} \alpha$ a valuable, although rather complicated, spectral line to study diverse atmospheric layers. Apart from the fact that there is photospheric contribution in both wing positions, their different behaviour is evident by the different pattern in the $2 \mathrm{D}$ maps shown in Fig. 1: the DS at $\pm 0.70 \AA$ at the IN shows impulsive brightenings (upwards motions) and darkenings over a largely uniform background. The $\pm 0.35 \AA \mathrm{DS}$, on the other hand, shows a more complicated pattern of upwards and downwards motions, of chromospheric origin. Concerning the canopy area, this different behaviour is justified by the power maps in 3 and $5 \mathrm{~min}$ ( 5.5 and $3.3 \mathrm{mHz}$, respectively) presented in Kontogiannis et al. (2010a,b), where a power halo around the network boundary at the $\pm 0.70 \AA \mathrm{DS}$ gives its place to a magnetic shadow at the $\pm 0.35 \AA$ AS. Having concluded that the two DSs show two distinct regions, that is, below and above the magnetic canopy (Kontogiannis et al. 2014), we examine wave propagation at this region in comparison with the IN (Fig. 3 right and left panels, respectively).

At the IN, throughout the entire frequency range, phase differences are positive, meaning that the deeper formed DS leads the higher formed one. Overall, the distribution does not represent dispersion relation typical of wave propagation (monotonically increasing with frequency). This is a common finding when comparison between chromospheric lines is made (Mein \& Mein 1976; Schmieder 1979; Fleck 1989). Similar to these studies, small positive phase differences are measured. The distribution is thicker at $5.5 \mathrm{mHz}$, where crosspower extends to higher positive phase differences, as a possible trail of acoustic wave 
propagation up to chromospheric layers. According to Fleck \& Schmitz (1991), the chromosphere undergoes resonant excitation at $3 \mathrm{~min}$ as a result of the longer period $p$-mode spectrum. Higher frequency oscillations, on the other hand, vanish at the chromosphere owing to the process of shock overtaking (Fleck \& Schmitz 1993), and transfer their energy to the 3 min range. This is a purely hydrodynamic process that could take place at the IN. Furthermore, the higher the frequency, the easier for the acoustic waves to undergo conversion to fast magneto-acoustic waves and reflect at low-lying canopies, which are also present at the IN (Nutto et al. 2012). This process might explain detection of negative phase differences above $5 \mathrm{mHz}$ (as a result of downwards propagating waves) and the decline of the high-frequency phase differences towards zero, which has also been seen in TRACE observations at UV continua (Krijger et al. 2001).

At the canopy (Fig. 3, right panel), the behaviour of phase differences is different. Negative values are measured up to $3.5 \mathrm{mHz}$, which means that on long timescales, the $\mathrm{H} \alpha \pm 0.35 \AA$ DS leads the $\mathrm{H} \alpha \pm 0.70 \AA \mathrm{DS}$. It is possible that there is an impact of the stochastic appearance-disappearance of mottles on the low-frequency behaviour. In our previous studies, we raised concerns about attributing the $7 \mathrm{~min}(2.4 \mathrm{mHz})$ period to representing acoustic oscillations. In Kontogiannis et al. (2014), we showed that the $7 \mathrm{~min}$ power at the chromosphere shows a different behaviour than that at the $5 \mathrm{~min}$, and it is very possible that it is not entirely due to magneto-acoustic waves. Conversely, negative phase differences at the low-frequency range may also be attributed to gravity waves (Mihalas \& Toomre 1981), which have been detected at the upper photosphere (Severino et al. 2003; Rutten \& Krijger 2003). If these gravity waves exist, they may very easily be affected by the magnetic field of the canopy and convert to either downwards slow magneto-acoustic waves or Alfvén waves (Newington \& Cally 2010).

In the $3-4 \mathrm{mHz}$ frequency range there is a steep increase of phase differences, and the distribution is thicker, with crosspower extending up to higher positive values. This may be explained on the grounds of a significant reduction of the cut-off frequency as a result of waves propagating along inclined magnetic field lines. These low-frequency magneto-acoustic waves are able to propagate upwards in the low- $\beta$ regime, as slow waves through "magneto-acoustic portals", which are created by strong and significantly inclined magnetic fields (Jefferies et al. 2006). Above the cut-off frequency, phase differences are lower than at the IN and phase differences become equal to $0^{\circ}$ up to $8 \mathrm{mHz}$. This is in total agreement with the results of Nutto et al. (2012), which indicate that the fast magneto-acoustic waves undergo reflection at the canopy and propagate almost horizontally, causing the adjacent atmospheric layers to oscillate in phase. There is also significant crosspower at negative phase differences, which is consistent with the phase difference maps presented in Kontogiannis et al. (2010a) and have been attributed to downwards propagating waves, after reflection on the magnetic canopy.

\subsection{Phase differences between $\mathrm{H} \alpha$ Doppler signal and intensity pairs}

Phase differences between intensity (as a proxy of temperature) and velocity may be used to infer the properties of acoustic waves. It is known from the literature that in running waves, temperature and velocity should be largely in phase, while for standing waves, intensity leads velocity by $90^{\circ}$ (adiabatic limit). When heat losses are rapid enough and also in the isothermal case, this phase difference may reach up to $180^{\circ}$ (Whitney 1958; Holweger \& Testerman 1975). Negative phase differences between $-180^{\circ}$ and $-90^{\circ}$ indicate downwards propagation.

We use these guidelines to interpret phase differences between the $\mathrm{H} \alpha$ average wing intensities and DS at $\pm 0.35 \AA$ and $\pm 0.70 \AA$; shown in Fig. 4 . The DS is not the actual velocity, but a parametric representation of velocity, calculated by intensity values. Regarding phase differences between average wing intensities and DS, crosstalk could be introduced, as shown by Moretti \& Severino (2002). These authors calculate for their data an offset up to $10^{\circ}$. Furthermore, $\mathrm{H} \alpha$ line formation processes complicate the interpretation of phase differences, since the dependence of the intensity on temperature, established at the outer wings of the line, weakens towards the line centre (Leenaarts et al. 2012). Finally, regarding ground-based observations, it has been reported that seeing may produce phase differences close to $0^{\circ}$ or $180^{\circ}$ at the high-frequency end of the distribution with increased coherence (Endler \& Deubner 1983). To see whether there is an influence from seeing, we examined the rest of the crosspower distributions more carefully, before normalization, and found that that there is little or no crosspower concentrated around $180^{\circ}$. Therefore, we conclude that the signal in the phase differences between the $\mathrm{H} \alpha$ DS and average wing intensity is not due to seeing. In fact, as already mentioned, comparisons of phase differences between $\mathrm{Ca}$ II H and $G$-band taken from the ground with results based on (seeing-free) observations from space (Lawrence \& Cadavid 2012) show remarkable agreement.

Keeping in mind these caveats, how can the measured phase differences be explained? In Fig. 4, positive phase difference means that the intensity leads upwards velocity and vice versa. The distributions at all occasions are concentrated around the $\pm 180^{\circ}$ line, indicating that velocity and intensity are largely in anti-phase, but there is also significant crosspower at the positive and negative phase difference domains. Phase differences at low and higher frequencies, reaching down to $90^{\circ}$ may correspond to the evanescent low-frequency waves and also the standing wave pattern of fast waves found around the canopy (Nutto et al. 2012). On the other hand, negative phase differences, between $-90^{\circ}$ and $-180^{\circ}$, correspond to downwards propagating waves. This could be the case for at least part of the crosspower detected in Fig. 4, since the process of conversion and transmission predicts the existence of fast waves directed towards the photosphere. We cannot exclude this possibility, even more since this behaviour is found at frequencies higher than $5 \mathrm{mHz}$.

Can we attribute the smaller differences found at the phase differences in the canopy regions to the interaction of the acoustic waves with the magnetic field? Kostik \& Khomenko (2013), examining a facular area, attribute the scatter of the phase differences throughout the entire range between $-180^{\circ}-180^{\circ}$ to the presence of the magnetic field, while Lites et al. (1982) also observe lower phase differences in V-I pairs at the network than at the IN (which appears to be the case in the right panels of Fig. 4). Overall, the phase difference distributions resemble those of Kulaczewski (1992) and Deubner et al. (1996) who used chromospheric lines, such as NaD1. Deubner et al. state that distributions, such as those observed in Fig. 4, may be explained on the grounds of the superposition of running and standing waves. Having measured the height of reflection of the fast waves and explained the observed oscillatory power on the grounds of mode conversion/transmission (Kontogiannis et al. 2014), we believe that the distributions in Fig. 4 support this scenario. However, as mentioned above, these results should be treated with caution. 

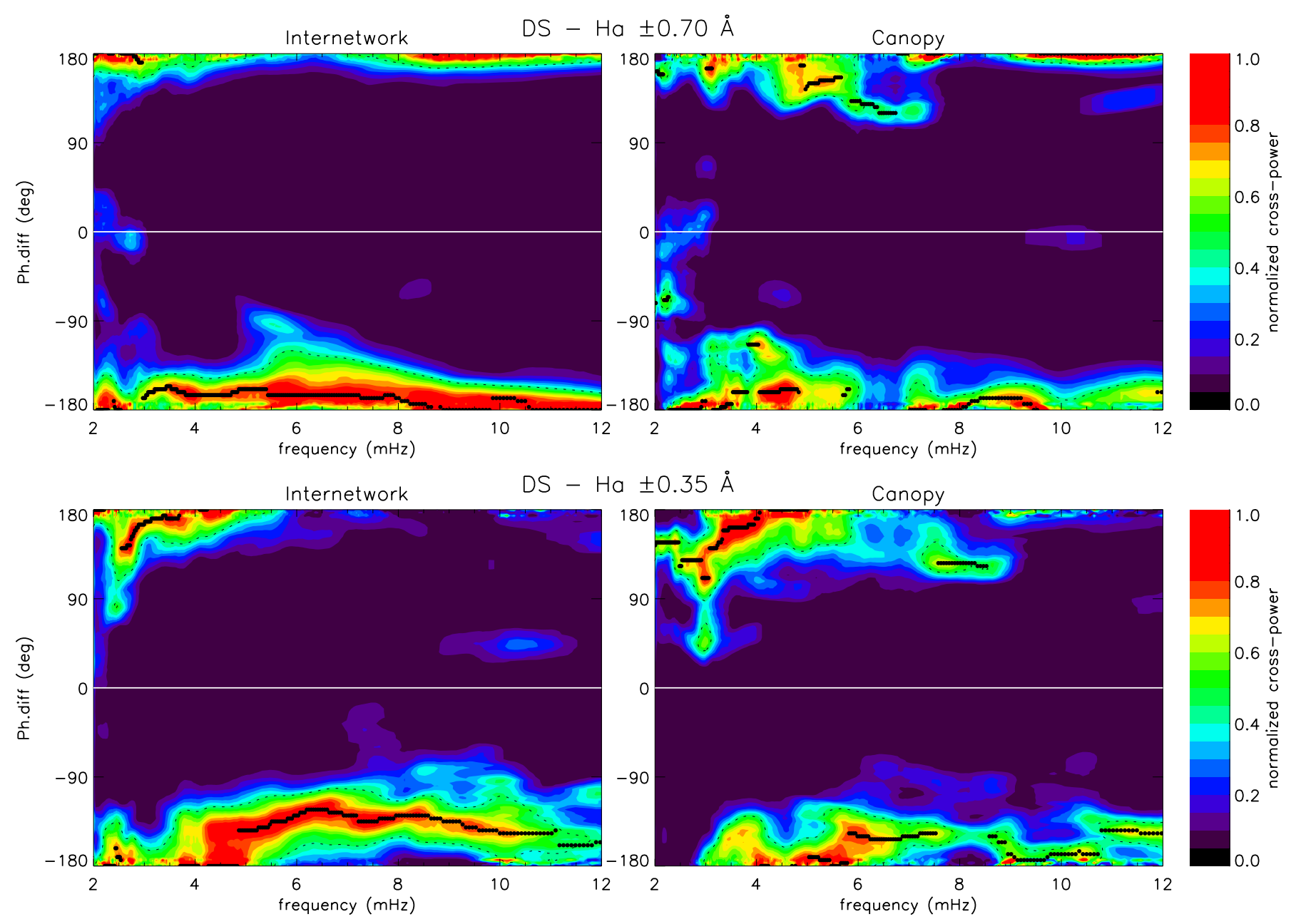

Fig. 4. Same as Fig. 2, but for $\mathrm{H} \alpha \mathrm{DS}$ and average wing intensity at $\pm 0.70 \AA$ (top) and $\mathrm{H} \alpha \mathrm{DS}$ and average wing intensity at $\pm 0.35 \AA$ (bottom).
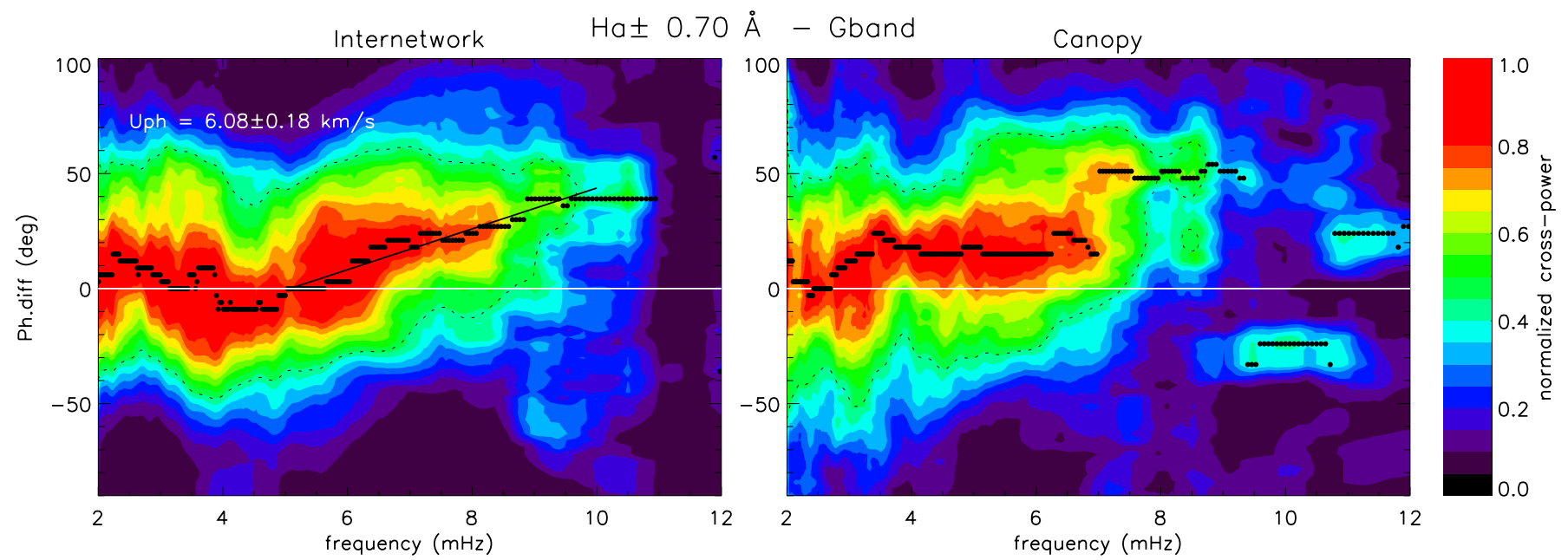

Fig. 5. Same as Fig. 2, but for $\mathrm{H} \alpha \pm 0.70 \AA-G$-band (top). A height separation of $150 \mathrm{~km}$ is assumed.

\subsection{Phase differences between $\mathrm{H} \alpha$ and $\mathrm{G}$-band}

Next, we examine phase differences between the $G$-band and the average $\mathrm{H} \alpha$ wing intensity at $\pm 0.70 \AA$ from the line centre (Fig. 5). The $G$-band shows details of the granulation, which is also dominant in the $\mathrm{H} \alpha$ outer wings along with strongly Doppler-shifted absorption features. This fact complicates the comparison with this intensity, introducing noise and reducing coherence, however, a coherent signal shows signs of wave propagation.

At the IN (Fig. 5, left panel), phase differences are concentrated around $0^{\circ}$ providing evidence of evanescent waves up to $6 \mathrm{mHz}$. Then phase differences increase, up to $10 \mathrm{mHz}$. Assuming a minimum height separation of $150 \mathrm{~km}$, the phase speed at the IN is $6.08 \pm 0.18 \mathrm{~km} \mathrm{~s}^{-1}$, a reasonable value for the acoustic speed at the photosphere and consistent with the 

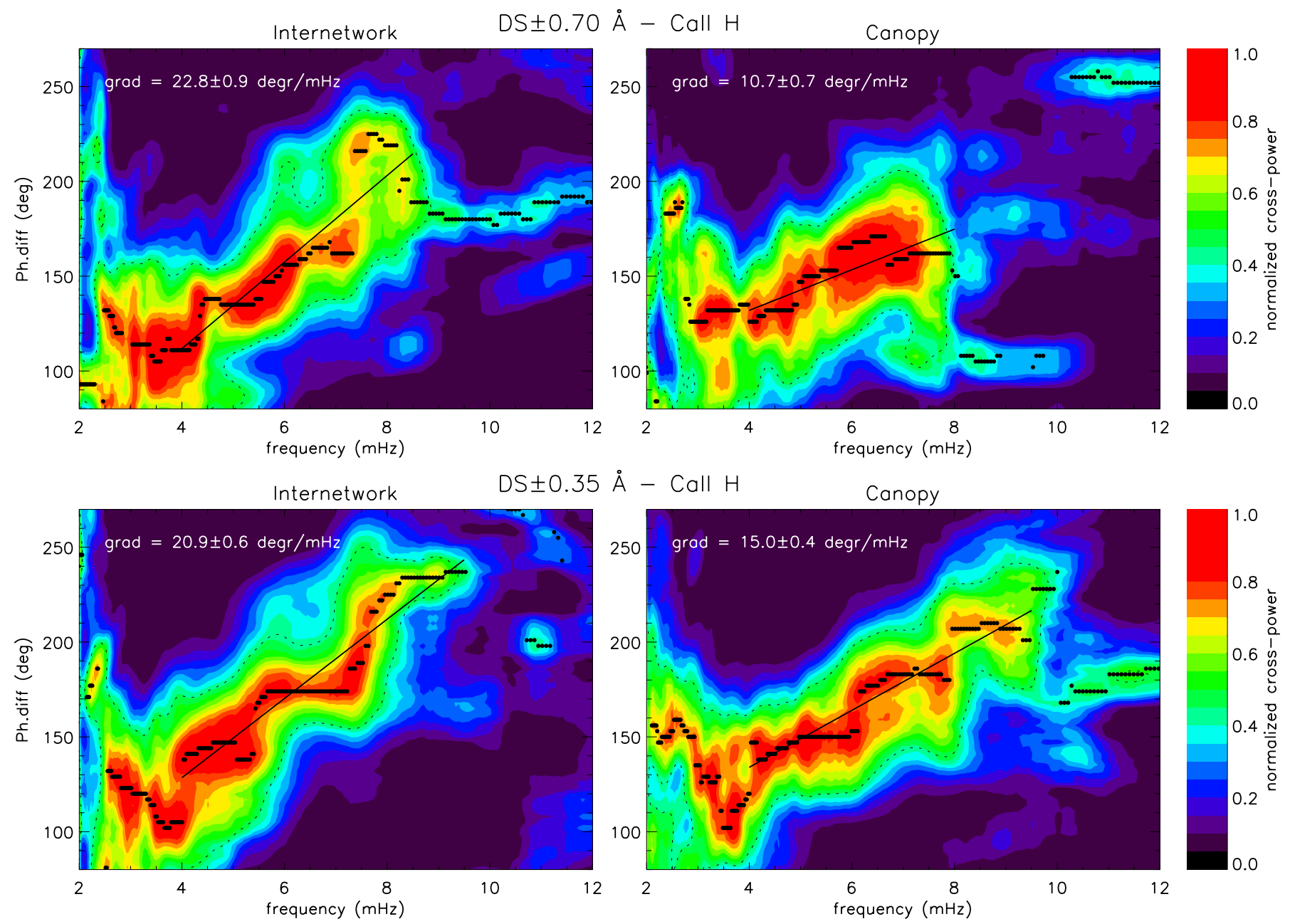

Fig. 6. Same as Fig. 2, for H $\alpha$ DS at $\pm 0.70 \AA$ and Ca II H intensity (top row) and H $\alpha$ DS at $\pm 0.35 \AA$ and Ca II H intensity (bottom row).

corresponding phase speed presented in Fig. 2. At the canopy, phase differences already start to increase at $3 \mathrm{mHz}$, showing the effect of $p$-mode leakage. Then, there is a plateau up to $7 \mathrm{mHz}$, above which the crosspower decreases significantly, although some higher positive and negative phase differences are measured. No regression line was plotted since there is no monotonic increase. From the shape of the distribution, it appears that the interaction with the magnetic canopy affects the phase differences in a manner similar to the $\mathrm{Ca}$ II $\mathrm{H}-G$-band pair, but more dramatically owing to the fact that the $\mathrm{H} \alpha \pm 0.70 \AA$ forms below the canopy (Kontogiannis et al. 2010b).

\subsection{Phase differences between $\mathrm{H} \alpha$ Doppler shifts and $\mathrm{Ca} \| \mathrm{H}$ intensities}

In phase difference analysis, velocity and intensity pairs from the same spectral line are usually compared, although in some cases, a comparison of velocities and intensities from different spectral regions has been used (Cram 1978; Judge et al. 2001). Motivation to perform a comparison between the Ca II $\mathrm{H}$ intensity and $\mathrm{H} \alpha$ DS came from inspecting the $\mathrm{H} \alpha$ DS time series along with the $\mathrm{Ca}$ II $\mathrm{H}$ intensity images. Localized, short duration, upwards motions are detected abundantly at the IN in $\mathrm{H} \alpha$ DS time series, which are reminiscent of the pattern of the intensity variations in $\mathrm{Ca} I \mathrm{H}$, superposed on the reversed granulation pattern. According to Rutten et al. (2008), the $\mathrm{H} \alpha$ wings show a response to the IN acoustic shocks that are abundant in the CaIIH. To check the response of the H $\alpha$ DS to the acoustic waves found at the upper photosphere, we constructed the halftone images of Fig. 6.

In all panels, phase differences exhibit a monotonic increase between $4-8 \mathrm{mHz}$ starting above $100^{\circ}-120^{\circ}$. Through observations and theory (Lites et al. 1993; Skartlien et al. 1994) it has been found that the $\mathrm{Ca}$ II H intensity leads the velocity in the same line by $100^{\circ}-120^{\circ}$ at the acoustic range. Based on this result, one might assume that a comparison between the $\mathrm{Ca}$ II H velocity and the $\mathrm{H} \alpha$ DS would give a similar distribution starting at $0^{\circ}$. Therefore, we conclude that there is acoustic wave propagation at the IN between the levels where $\mathrm{Ca}$ II H and $\mathrm{H} \alpha$ DS are formed. Given the fact that the IN is dominated by acoustic shocks, as suggested by Carlsson \& Stein (1997), this provides an observational evidence of the $\mathrm{H} \alpha$ velocity response to these IN acoustic shocks.

This wave propagation (monotonic increase in phase difference) commences at frequencies lower than the typical acoustic cut-off. Furthermore, the gradient of the distribution of the $\pm 0.70 \AA$ DS-CaIIH is slightly higher than the $\pm 0.35 \AA$ DS-Ca II H pair, which is consistent with a larger height separation in the former case than in the latter. However, the similar values of the gradients demonstrate that this difference in height separation does not contribute dramatically to the measured phase differences, which is in agreement with the small phase differences presented in Fig. 3 between the two DS of $\mathrm{H} \alpha$. At the magnetic canopy, these gradients are even lower, which is 
also consistent with the reflection of the fast magneto-acoustic waves near the network boundaries.

A comparison between high temporal, spatial, and spectral resolution observations in $\mathrm{H} \alpha$ and $\mathrm{Ca} I \mathrm{H}$, which are becoming increasingly available to the community, will lead to valuable results on the connection between the acoustically dominated upper photosphere/lower chromosphere at the temperature minimum region and the $\mathrm{H} \alpha$ line formation at the IN.

\section{Conclusions and discussion}

We have examined phase differences of oscillations using time series of co-temporal photospheric and chromospheric images of a solar quiet region. We conclude that the small-scale magnetic field of the quiet Sun affects the phase differences of oscillations in the various heights sampled by our data, in the context of magnetoacoustic mode conversion/transmission. This effect was studied in the past as "network-IN distinctions", but it now seems that the crucial distinction should be "canopy-IN". As already mentioned, the traditional view of the IN as a field-free component of the solar atmosphere is itself under revision in light of the latest high-resolution observations of the solar magnetism (Lites et al. 2008).

In most of the cases examined in this study, the acoustic cutoff frequency is lower than that expected for a gravitationally stratified atmosphere that is free of magnetic fields. Figures 2, 3, and 5 show evidence of wave propagation for frequencies as low as $3 \mathrm{mHz}$, at the canopy and in some cases at the IN, with a phase speed roughly equal to the local sound speed. The importance of this mechanism, as illustrated also in numerical studies, is the fact that slow waves are easily channelled to the upper atmospheric layers, travelling along the magnetic field lines.

Above the acoustic cut-off frequency, wave propagation is detected between $G$-band - CaII H, $G$-band - $\mathrm{H} \alpha$ and $\mathrm{H} \alpha$ DS - Ca IIH. The presence of the magnetic canopy appears to alter wave propagations by systematically lowering the gradient of the phase difference distributions, hence, increasing the phase speed. In our context, this is an indication of wave energy being transferred to the fast magneto-acoustic mode, combined with the superposition of a standing wave pattern on the existing acoustic wave field. This idea has been speculated in the past by Deubner \& Fleck (1990), but their data analysis lacked simultaneous high-resolution magnetograms. Kontogiannis et al. (2014), based on the dependence of the oscillatory power on the magnetic field inclination, demonstrated that mode conversion and transmission takes place at the magnetic canopy. As already mentioned, conversion to fast magnetoacoustic waves is more efficient for higher frequencies and produces shorter phase differences due to their higher phase speed (fast speed). This may explain the flattening of the crosspower distribution in higher frequencies, at the magnetic canopy (Fig. 5), while the presence of small-scale and unresolved magnetic fields may be invoked to explain this effect, even at the undisturbed IN where acoustic wave propagation does not seem to persist for high frequencies. In the context of the proposed mechanism, the magnetic field acts as a directional filter, which eventually allows only acoustic disturbances travelling along the magnetic field lines. As for the energy carried by the fast waves, in the 3D case it has been shown that Alfvén waves are produced at the sites of reflection (Khomenko \& Cally 2012). We believe that our measurements of phase differences between intensity and DS in $\mathrm{H} \alpha$ support this scenario, but this specific subject should be revisited using several high-resolution, ground-based observations of the chromosphere.
Our analysis also reveals that the $\mathrm{H} \alpha$ DS exhibits a response to the phenomena occurring in the temperature minimum region, which is sampled by the Ca II H line. Leenaarts et al. (2012) have shown that the $\mathrm{H} \alpha$ line is decoupled from temperature, above $1 \mathrm{Mm}$ and is sensitive to density variations. Acoustic shocks produce such enhancements in density (Carlsson \& Stein 1997) at the IN and mostly below the magnetic canopy and lead to DS variations in $\mathrm{H} \alpha$. These acoustic shocks have been found to avoid magnetic field concentrations at the IN and the network (Vecchio et al. 2009), and magnetic shadows are an example of this phenomenon. The effect of mode conversion and transmission is also visible here, as discussed in the previous section.

When interpreting results of phase difference analysis, difficulties arise from the width in the contribution functions of the bandpasses used, the vagueness of the HOF relating to the corresponding physical and magnetic conditions, and the uncertainty/variety of the solar conditions across the FOV. In this study, we assumed some average values for the height separation of the layers sampled by our data based on the estimated formation heights of $\mathrm{H} \alpha$ (Leenaarts et al. 2006) and $\mathrm{Ca}$ II H (Rutten et al. 2004b). Non-LTE effects involved in the formation of $\mathrm{H} \alpha$ along with the limited spectral coverage of the $\mathrm{H} \alpha$ profile in our data does not allow us to completely disentangle the contribution of the line width, opacity, and line shift. Therefore, results based on the $\mathrm{H} \alpha$ line should be treated with caution, particularly those concerning the interpretation of V-I phase spectra. These spectra are even more complicated taking into account that the determination of the average wing intensities and DS may introduce crosstalk between velocity and intensity signals (Moretti \& Severino 2002). This is something worth revisiting in the future in light of improved spectral and temporal resolution chromospheric observations.

To our defence and as far as the validity of our approach is concerned, the confirmation of previous results concerning the Ca II H-G-band intensity pair (Rutten et al. 2004b; Lawrence \& Cadavid 2012) provides assurance that the determination of phase differences itself, through our approach, is accurate. There are, however, limitations in our method: the oblique propagation of slow and fast waves (along and perpendicular to the magnetic field) imposes a selection effect in favour of vertically propagating disturbances. In order to fully identify the relation between oscillations at the lower and upper parts of the chromosphere, one must be able to combine large statistical significance (e.g. as in de Wijn et al. 2009) with a localized treatment that may highlight the effect of the diversity of the magnetic field configuration, taking the magnetic field inclination into account, for example (in a manner similar to the work of Bloomfield et al. 2007). Admittedly, inside the canopy area, the inclination varies, since the magnetic field is largely vertical near the network and progressively more inclined towards the outer parts of the canopy. Since the transmission/conversion of magneto-acoustic waves depends on the inclination of the magnetic field, an examination of the phase differences variation in regions of different inclination angles would be ideal. However, it is difficult to achieve a statistically significant result in the fine magnetic concentrations of the network, as opposed to the extended active regions. Data sets of several network areas would be required, including simultaneous high-resolution magnetograms to amass a large statistical sample. Our analysis demonstrates the overall effect of the magnetic canopy on the wave propagation, but we believe that analysis of higher spectral and spatial resolution data will shed more light on the propagation of waves through the magnetized chromospheric plasma. 
Acknowledgements. The observations have been funded by the Optical Infrared Coordination network (OPTICON, http://www.ing.iac.es/opticon), a major international collaboration supported by the Research Infrastructures Program of the European Commission's sixth Framework Program. The research was funded through the project "SOLAR-4068", which is implemented under the "ARISTEIA II" Action of the operational program "Education and Lifelong Learning" and is cofunded by the European Social Fund (ESF) and Greek national funds. The DOT was operated at the Spanish Observatorio del Roque de los Muchachos of the Instituto de Astrofísica de Canarias. The authors thank P. Sütterlin for the DOT observations and R. Rutten for the data reduction. Hinode is a Japanese mission developed and launched by ISAS/JAXA, collaborating with NAOJ as a domestic partner, and NASA and STFC (UK) as international partners. Scientific operation of the Hinode mission is conducted by the Hinode science team organized at ISAS/JAXA. This team mainly consists of scientists from institutes in the partner countries. Support for the post-launch operation is provided by JAXA and NAOJ (Japan), STFC (UK), NASA, ESA, and NSC (Norway). Hinode SOT/SP Inversions were conducted at NCAR under the framework of the Community Spectro-polarimetric Analysis Center (CSAC; http://www.csac.hao.ucar.edu).

\section{References}

Beckers, J. M. 1968, Sol. Phys., 3, 367

Bel, N., \& Leroy, B. 1977, A\&A, 55, 239

Bloomfield, S. D., McAteer, J. R. T., Lites, B. W., et al. 2004, ApJ, 617, 623

Bloomfield, D. S., Lagg, A., \& Solanki, S. K. 2007, ApJ, 671, 1005

Bogdan, T. J., Carlsson, M., Hansteen, V., et al. 2003, ApJ, 599, 626

Cally, P. S. 2007, Astron. Nachr., 328, 286

Carlsson, M., \& Bogdan T. J. 2006, Philos. Trans. R. Soc. London A, 364, 395

Carlsson, M., \& Stein, R. F. 1997, ApJ, 481, 500

Cram, L. E. 1978, A\&A, 70, 345

De Wijn, A. G., McIntosh, S. W., \& De Pontieu, B. 2009, ApJ, 702, L168

Deubner, F.-L. 1975, A\&A, 44, 371

Deubner, F.-L., \& Fleck, B. 1989, A\&A, 213, 423

Deubner, F.-L., \& Fleck, B. 1990, A\&A, 228, 506

Deubner, F.-L., Ulrich, R. K., \& Rhodes, E. J. Jr. 1979, A\&A, 72, 177

Deubner, F.-L., Fleck, B., Marmolino, C., \& Severino, G. 1990, A\&A, 236, 509

Deubner, F.-L., Waldschik, T., \& Steffens, S. 1996, A\&A, 307, 936

Endler, F., \& Deubner, F.-L. 1983, Sol. Phys., 121, 291

Fleck, B., \& Deubner, F.-L. 1989, A\&A, 224, 245

Fleck, B., \& Schmitz, F. 1991, A\&A, 250, 235

Fleck, B., \& Schmitz, F. 1993, A\&A, 273, 671

Gabriel, A. H. 1976, Roy. Soc. Phul. Trans. London, 281, 339

Holweger, H., \& Testerman, L. 1975, Sol. Phys., 43, 271

Jefferies, S. M., McIntosh, S. W., Armstrong, J. D., et al. 2006, ApJ, 648, L151

Judge, P. G., Tarbell, T. D., \& Wilhelm, K. 2001, ApJ, 554, 424

Khomenko, E., \& Cally, P. S. 2012, ApJ, 746, 68

Khomenko, E., \& Collados, M. 2006, ApJ, 653, 739

Khomenko, E., \& Collados, M. 2009, A\&A, 506, L5

Khomenko, E., Collados, M., \& Felipe, T. 2008, Sol. Phys., 251, 589

Kneer, F., \& von Uexküll, M. 1985, A\&A, 144, 443

Kontogiannis, I., Tsiropoula, G., \& Tziotziou, K. 2010a, A\&A, 510, A41
Kontogiannis, I., Tsiropoula, G., Tziotziou, K., \& Georgoulis, M. K. 2010b, A\&A, 524, A12

Kontogiannis, I., Tsiropoula, G., \& Tziotziou, K. 2011, A\&A, 531, A66

Kontogiannis, I., Tsiropoula, G., \& Tziotziou, K. 2014, A\&A, 567, A62

Kostik, R., \& Khomenko, E. 2013, A\&A, 559, A107

Kosugi, T., Matsuzaki, K., Sakao, T., et al. 2007, Sol. Phys., 243, 3

Krijger, J. M., Rutten, R. J., Lites, B. W., et al. 2001, A\&A, 379, 1052

Kulaczewski, J. 1992, A\&A, 261, 602

Lawrence, J. K., \& Cadavid, A. C. 2010, Sol. Phys., 261, 35

Lawrence, J. K., \& Cadavid, A. C. 2012, Sol. Phys., 280, 125

Leenaarts, J., Rutten, R. J., Sütterlin, P., et al. 2006, A\&A, 449, 1209

Leenaarts, J., Carlsson, M., \& Rouppe Van Der Voort, L. 2012, ApJ, 749, 136

Leighton, R. B., Noyes, R. W., \& Simon, G. W. 1962, ApJ, 135, 474

Lites, B. W., \& Chipman, E. G. 1979, ApJ, 231, 570

Lites, B. W., Chipman, E.G ., \& White, O. R. 1982, ApJ, 253, 367

Lites, B. W., Rutten, R. J., \& Kalkofen, W. 1993, ApJ, 414, 345

Lites, B. W., Kubo, M., Socas-Navarro, H., et al. 2008, ApJ, 672, 1237

McIntosh, S. W., \& Judge, P. G. 2001, ApJ, 561, 420

McIntosh, S. W., \& Smillie, D. G. 2004, ApJ, 604, 924

McIntosh, S. W., Fleck, B., \& Judge, P. G. 2003, A\&A, 405, 769

Michalitsanos, A. G. 1973, Sol. Phys., 30, 47

Mihalas, B. W., \& Toomre, J. 1981, ApJ, 249, 349

Mein, N., \& Mein, P. 1976, Sol. Phys., 49, 231

Moretti, P. F., \& Severino, G. 2002, A\&A, 384, 638

Newington, M. E., \& Cally, P. S. 2010, MNRAS, 402, 386

Nutto, C., Steiner, O., \& Roth, M., 2012, A\&A, 542, L30

Reardon, K. P., Uitenbroek, H., \& Cauzzi, G. 2009, A\&A, 500, 1239

Rosenthal, C. S., Bogdan, T. J., Carlsson, M., et al. 2002, ApJ, 564, 508

Rutten, R. J., \& Krijger, J. M. 2003, A\&A, 407, 735

Rutten, R. J., Hammerschlag, R. H., Bettonvil, F. C. M., et al. 2004a, A\&A, 413, 1183

Rutten, R. J., de Wijn, A. G., \& Sütterlin, P. 2004b, A\&A, 416, 333

Rutten, R. J., van Veelen, B., \& Sütterling, P. 2008, Sol. Phys., 251, 533

Schmieder, B. 1979, A\&A, 74, 273

Schunker, H., \& Cally, P. S. 2006, MNRAS, 372, 551

Severino, G., Oliviero, M,. Straus, Th., \& Ulrich, R. K. 2003, Mem. Sci. Astron. It., 74, 595

Skartlien, R., Carlsson, M., \& Stein, R. F. 1994, in Proc. Chromospheric Dynamics, ed. M. Carlsson, 79

Stangalini, M., Del Moro, D., Berrilli, F., \& Jefferies, S. M. 2011, A\&A, 534, A65

Suematsu, Y. 1990, Lect. Notes Phys., 367, 211

Torrence, C., \& Compo, G. P. 1998, Bull. Am. Meteor. Soc., 79, 61

Tsiropoula, G., Tziotziou, K. Schwartz, P., \& Heinzel, P. 2009, A\&A, 493, 217

Tsiropoula, G., Tziotziou, K., Kontogiannis, I., et al. 2012, Space Sci. Rev., 169, 181

Tsuneta, S., Suematsu, Y., Ichimoto, K., et al. 2008, Sol. Phys., 249, 167

Tziotziou, K., Tsiropoula, G., \& Mein, P. 2004, A\&A, 423, 1133

Vecchio, A., Cauzzi, G., Reardon, K. P., et al. 2007, A\&A, 461, L1

Vecchio, A., Cauzzi, G., Reardon, K. P. 2009, A\&A, 494, 269

Vernazza, J. E., Avrett, E. H., \& Loeser, R. 1981, ApJSS, 45, 635

Whitney, C. A. 1958, Smithsonian Contributions to Astrophysics, 2, 365

Worrall, G. 2002, MNRAS, 335, 628 